\title{
A LATTICE-POINT PROBLEM
}

\author{
BY \\ BURTON RANDOL(1),(2)
}

Introduction. Suppose $n \geqq 2$, and suppose $T\left(z_{1}, \cdots, z_{n}\right)=T(Z)$ is a positive function, defined in $E^{n}-\{0\}$ and $C^{\infty}$ there, and homogeneous of positive weight $w$, i.e., $T(r Z)=r^{w} T(Z)(r>0)$. (The differentiability restriction on $T(Z)$ is simply one of convenience. It can generally be weakened to suit the particular case at hand.)

Define

1. $C=\{Z \mid T(Z) \leqq 1\}$.

2. $\partial C=\{Z \mid T(Z)=1\}$.

3. $V=\int_{C} d V_{Z} \quad\left(d V_{Z}=d z_{1} \cdots d z_{n}\right)$.

4. $N(x)=$ the number of integral lattice-points $N$, such that $T(N) \leqq x$ $(x>0)$.

5. $R(x)=N(x)-V x^{n / w}$.

The problem of the title consists of finding asymptotic estimates for $R(x)$, and in $\$ 1$, we will obtain a general theorem along these lines, assuming a minimum amount of knowledge about $\partial C$. In $\S 2$, we will indicate by an example how in some cases the results of $\S 1$ can be considerably refined. The case in which $\partial C$ has everywhere positive Gaussian curvature is discussed in [2] and [3].

1. We begin with a postulate about $\partial C$. Several cases in which the postulate is valid are given in [2], [3], and [6]. It is, moreover, valid in many arithmetically interesting cases which are not treated in these papers. For example, if $T(Z)=z_{1}^{2 k}+\cdots+z_{n}^{2 k}$, it is not difficult to show that the numbers $\alpha$ and $h$ which occur in the postulate can be taken to be $(n-1) / 2 k$, and $[(n-1) / 2 k]+1$, respectively.

Postulate. There exists a positive integer $h$, and a number $0<\alpha \leqq(n-1) / 2$, such that for any function $f(Z), C^{h}$ in a neighborhood of $\partial C$, there is a positive number $M(f)$, for which

$$
\left|\int_{\partial C} f(Z) e^{2 \pi i(Y, Z)} d S_{Z}\right| \leqq M(f)|Y|^{-\alpha} .
$$

Received by the editors May 11, 1965.

(1) This research was partially supported by the National Science Foundation under Grant No. NSF-GP-1814.

(2) It has come to my attention that van der Corput has obtained, by different methods, the estimate we derive for the example in \$2. As we shall show in a subsequent paper, the techniques illustrated in $\$ 2$ generalize without difficulty to several dimensions. 
Furthermore, $M(f)$ depends only on bounds for $f(Z)$ and its first $h$ derivatives in a neighborhood of $\partial C$. (Here $Y=\left(y_{1}, \cdots, y_{n}\right),|Y|=\left(y_{1}^{2}+\cdots+y_{n}^{2}\right)^{1 / 2}$, and $d S_{\mathrm{Z}}$ is the area element on $\partial C$.)

We can now state the result of $\S 1$, which consists of an upper bound for $|R(x)|$.

THEOREM. $R(x)=O\left(x^{R}\right)$, where $R=\left(n^{2}-n-n \alpha\right) / w(n-\alpha)$.

REMARK. In the proof of this theorem, it will be convenient to make the assumption that $w \geqq w_{0}=[n-\alpha]+h+2$, and we shall henceforth assure that this is the case. This involves no loss of generality, since if $w<w_{0}$, we can obtain the desired result by replacing $T(Z)$ by $(T(Z))^{w_{0} / w}$, and then replacing $x$ by $x^{w_{0} / w}$ in the resulting estimate.

We now pass to the proof of the theorem, which, while not difficult, is somewhat long, and for the sake of clarity, we state now without proof two preliminary lemmas. We will show how the theorem follows from these, and then prove the lemmas themselves. In order to state the lemmas, we need a few definitions.

Define

6. $J(x, Z)=$ the characteristic function of the set $\{Z \mid T(Z) \leqq x\}$.

7. $\mathcal{J}(x, Y)=$ the Fourier transform of $J(x, Z)$ in the second variable. I.e.,

$$
\hat{J}(x, Y)=\int_{E^{n}} J(x, Z) \exp [2 \pi i(Y, Z)] d V_{Z} .
$$

8. $r_{1}=(n w-n-w \alpha) / w(n-\alpha)$.

9. $r_{2}=\alpha / w(n-\alpha)$.

10. $z=x^{r_{1}}$.

11. $p=[n-\alpha]$.

12. For each positive integer $q$, and function $f(x)$, integrable on finite subintervals of $(0, \infty)$, set

$$
I^{q} f(x)=\int_{x}^{x+z} d x_{1} \cdots \int_{x_{q}-1}^{x_{n-1}+z} f\left(x_{q}\right) d x_{q} \quad\left(x_{0}=x\right) .
$$

For $q=0$, define $I^{0} f(x)=f(x)$. Then for each $q, I^{q}$ is a linear operator. If $g$ is a function of several variables, we attach a subscript to denote the variable in which the operator is applied. E.g.,

$$
I_{x}^{q} g(x, y)=\int_{x}^{x+z} d x_{1} \cdots \int_{x_{n-1}}^{x_{n-1}+z} g\left(x_{q}, y\right) d x_{q} .
$$

LEMMA 1. $I_{x}^{p} \hat{J}(x, Y)=O\left(F_{1}(x, Y)\right)$, where

$$
F_{1}(x, Y)=x^{p r_{1}+(n-1-\alpha) w^{-1}}|Y|^{-(1+\alpha)},
$$

and the estimate is uniform in $X$ and $Y(Y \neq 0)$, 
Lemma 1 furnishes an estimate which is good in $x$ but not in $Y$. Lemma 2 goes the other way around.

LEMMA 2. $I_{x}^{p} \hat{J}(x, Y)=O\left(F_{2}(x, Y)\right)$, where

$$
F_{2}(x, Y)=x^{(n+p w-\alpha-p-1) w^{-1}}|Y|^{-(1+p+\alpha)},
$$

and the estimate is uniform in $x$ and $Y(Y \neq 0)$.

Proof of the theorem. To begin with, it is clear that $N(x)=\Sigma J(x, N)$, where the summation is over all integral lattice-points.

For each $\varepsilon>0$, let $\delta_{\varepsilon}(Z)$ be a nonnegative $C^{\infty}$ function with support in the ball $|Z| \leqq \varepsilon$, and such that

$$
\int_{E^{n}} \delta_{\imath}(Z) d V_{Z}=1
$$

Set $J_{\varepsilon}(x, Y)=\int_{E^{n}} J(x, Z) \delta_{\varepsilon}(Y-Z) d V_{Z}$, and $N_{\varepsilon}(x)=\sum J_{\varepsilon}(x, N)$.

Then for each fixed $\varepsilon$ and $x, J_{\varepsilon}(x, Y)$ is $C^{\infty}$ in $Y$ with compact support Moreover, for $x$ in bounded intervals, $N_{\varepsilon}(x)$ is uniformly bounded for small $\varepsilon$, and if $x$ is such that the set $\{Z \mid T(Z)=x\}$ contains no integral lattice-points (all but a countable number of $x$ 's have this property), then $N(x)=\lim _{\varepsilon \rightarrow 0} N_{\varepsilon}(x)$.

Now by the Poisson summation formula,

$$
\begin{aligned}
\Sigma J_{\varepsilon}(x, N) & =\Sigma \hat{J}_{\varepsilon}(x, N) \\
& =\Sigma \hat{\delta}_{\varepsilon}(N) \hat{J}(x, N) \\
& =\hat{\delta}_{\varepsilon}(0) \hat{J}(x, 0)+\Sigma^{\prime} \hat{\delta}_{\varepsilon}(N) \hat{J}(x, N) \\
& =V x^{n / w}+\Sigma^{\prime} \hat{\delta}_{\varepsilon}(N) \hat{J}(x, N),
\end{aligned}
$$

where the prime on a summation sign means that the origin is omitted from the sum.

But by the dominated convergence theorem,

$$
I^{p} N(x)=\lim _{\varepsilon \rightarrow 0} I^{p} N_{\varepsilon}(x),
$$

so

$$
\begin{aligned}
I^{p} N(x) & =I^{p} V x^{n / w}+\lim _{\varepsilon \rightarrow 0} I_{x}^{p}\left(\Sigma^{\prime} \hat{\delta}_{\varepsilon}(N) \hat{J}(x, N)\right) \\
& =V I^{p} x^{n / w}+\lim _{\varepsilon \rightarrow 0} \Sigma^{\prime} \hat{\delta}_{\varepsilon}(N) I_{x}^{p} \hat{J}(x, N),
\end{aligned}
$$

the interchange of summation and integration being justified by the rapidly decreasing character of $\hat{\delta}_{\varepsilon}(N)$.

Thus by Lemmas 1 and 2,

$$
I^{p} N(x)=V I^{p} x^{n / w}+\lim _{\varepsilon \rightarrow 0} \sum_{|N| \leqq x^{r_{2}}} \hat{\delta}_{\varepsilon}(N) O\left(F_{1}(x, N)\right)+\lim _{\varepsilon \rightarrow 0} \sum_{|N|>x^{r_{2}}} \hat{\delta}_{\varepsilon}(N) O\left(F_{2}(x, N)\right) .
$$


Now $\left|\hat{\delta}_{\varepsilon}(N)\right| \leqq 1$, so we see (e.g., by comparison with appropriate integrals) that both the last two limits are $O\left(x^{p r_{1}+R}\right)$. I.e., $I^{p} N(x)=V I^{p} x^{n / w}+O\left(x^{p r_{1}+R}\right)$. But $V I^{p} x^{n / w}=V z^{p} x_{0}^{n / w}$, for some $x_{0}$ between $x$ and $x+p z$, and by the meanvalue theorem,

I.e.,

$$
x_{0}^{n / w}-x^{n / w}=O\left(z(x+p z)^{(n / w)-1}\right)=O\left(z x^{(n / w)-1}\right) .
$$

$$
\begin{aligned}
V I^{p} x^{n / w} & =V_{g}^{p} x^{n / w}+O\left(z^{p+1} x^{(n / w)-1}\right) \\
& =V x^{(n / w)+p r_{1}} z^{p}+O\left(x^{p r_{1}+R}\right) .
\end{aligned}
$$

Combining this with our previous information, we find that

$$
I^{p} N(x)=V x^{(n / w)+p r_{1}}+O\left(x^{p r_{1}+R}\right) .
$$

Now $N(x)$ is nondecreasing, so $z^{p} N(x) \leqq I^{p} N(x) \leqq z^{p} N(x+p z)$. I.e.,

$$
x^{p r_{1}} N(x) \leqq V x^{(n / w)+p r_{1}}+O\left(x^{p r_{1}+R}\right) \leqq x^{p r_{1}} N\left(x+p x^{r_{1}}\right) .
$$

By the first half of this inequality, we find that $N(x) \leqq V x^{n / w}+O\left(x^{R}\right)$, and making the substitution $x_{1}=x+p x^{r_{1}}$, we easily obtain from the second half that $N\left(x_{1}\right) \geqq V x_{1}^{n / w}+O\left(x_{1}^{R}\right)$. I.e., $N(x)=V x^{n / w}+O\left(x^{R}\right)$, or $R(x)=O\left(x^{R}\right)$. q.e.d.

Proof of Lemma 1.

$$
\hat{J}(x, Y)=x^{n / w} \int_{C} \exp \left\{2 \pi i|Y| x^{1 / w}(\beta(Y), Z)\right\} d V_{Z},
$$

where $\beta(Y)=Y /|Y|$, and by the divergence theorem, this integral is equal to

$$
x^{(n-1) / w}(2 \pi i|Y|)^{-1} \int_{\partial C} \exp \left\{2 \pi i|Y| x^{1 / w}(\beta(Y), Z)\right\}(\beta(Y), n(Z)) d S_{Z},
$$

where $n(Z)$ is the outward normal to $\partial C$ at the point $Z$.

Now by our postulate, the integral in this last expression is $O\left(|Y|^{-\alpha} x^{-\alpha / w}\right)$, uniformly in $x$ and $Y$, since $(\beta(Y), n(Z))$ can be differentiably extended (say homogeneously of weight zero) to a neighborhood of $\partial C$, and the derivatives of the result are bounded independently of $Y$. Consider now $I_{x}^{p} \hat{J}(x, Y)$. The domain of integration of the iterated integral has measure $z^{p}=x^{p r_{1}}$, and since the argument of the integrand can only range between $x$ and $x+p z$, we immediately obtain that $I_{x}^{p} \hat{J}(x, Y)=O\left(F_{1}(x, Y)\right)$, uniformly in $x$ and $Y(Y \neq 0)$. q.e.d.

For the proof of Lemma 2, we require one additional lemma.

LEMma 3. Suppose $B(Z)$ is $C^{j}$ in $E^{n}$, for some $j \geqq 2$, and suppose $\beta$ is a unit vector. Then there exists a vector field $F(Z)=\left(f_{1}(Z), \cdots, f_{n}(Z)\right)$, such that

1. Each $f_{i}(Z)$ is (at least) of differentiability class $C^{j-1}$ in $E^{n}$.

2. $\operatorname{div} F(Z)=0$.

3. $(\beta, F(Z))=B(Z)$.

4. For each compact set $S_{1}$, there exists a compact set $S_{2}$, such that 
$f_{1}(Z), \cdots, f_{n}(Z)$, and their first $j-1$ derivatives on $S_{1}$, can be bounded in terms of $B(Z)$ and its first $j$ derivatives on $S_{2}$. The bound does not, however, depend on $\beta$.

Proof. Set $\beta=\left(\beta_{1}, \cdots, \beta_{n}\right)$. Then since $|\beta|=1$, at least one of the $\beta_{i}$ has absolute value $\geqq n^{-1 / 2}$. We will suppose $\beta_{1}$ has this property, since the proof proceeds along the same lines in the other cases.

Define $f_{i}(Z)=0$, for $3 \leqq i \leqq n$. The equations to be satisfied then become

$$
\begin{aligned}
\frac{\partial f_{1}}{\partial z_{1}}+\frac{\partial f_{2}}{\partial z_{2}} & =0 . \\
\beta_{1} f_{1}+\beta_{2} f_{2} & =B .
\end{aligned}
$$

Suppose the desired vector field existed. Then, differentiating (2) with respect to $z_{1}$, we would have

or

$$
\beta_{1} \frac{\partial f_{1}}{\partial z_{1}}+\beta_{2} \frac{\partial f_{2}}{\partial z_{1}}=\frac{\partial B}{\partial z_{1}}
$$

$$
\frac{\partial f_{1}}{\partial z_{1}}+\frac{\beta_{2}}{\beta_{1}} \frac{\partial f_{2}}{\partial z_{1}}=\frac{1}{\beta_{1}} \frac{\partial B}{\partial z_{1}} .
$$

Subtracting (1) from this, we get

$$
\left(\frac{\beta_{2}}{\beta_{1}} \frac{\partial}{\partial z_{1}}-\frac{\partial}{\partial z_{2}}\right) f_{2}=\frac{1}{\beta_{1}} \frac{\partial B}{\partial z_{1}} .
$$

If we set $K=\left(1+\left(\beta_{2} / \beta_{1}\right)^{2}\right)^{1 / 2}$, and divide both sides of the last equation by $K$, we get

$$
\frac{1}{K}\left(\frac{\beta_{2}}{\beta_{1}} \frac{\partial}{\partial z_{1}}-\frac{\partial}{\partial z_{2}}\right) f_{2}=\frac{1}{K \beta_{1}} \frac{\partial B}{\partial z_{1}} .
$$

The quantity on the left is simply the directional derivative of $f_{2}$ in the direction $d=\left(\beta_{2} / \beta_{1},-1,0, \cdots, 0\right)$. Let $P$ be the plane through the origin perpendicular to $d$. Then $f_{2}(Z)$ can be got (modulo a function which is constant on lines parallel to $d$ ) by integrating $\left(1 / K \beta_{1}\right)\left(\partial B / \partial z_{1}\right)$ from $P$ to $Z$ in a direction parallel to $d$. If, now, we simply define $f_{2}(Z)$ by this integral, and define $f_{1}(Z)$ to be $\beta_{1}^{-1}\left(B(Z)-\beta_{2} f_{2}(Z)\right.$ ), all the conclusions of the lemma are satisfied. q.e.d.

Definition. Suppose that $Y$ is a point in $E^{n}, y$ is a positive number, and $F(Z)=\left(f_{1}(Z), \cdots, f_{n}(Z)\right)$ is a continuous vector field in a neighborhood of $\partial C$. Define

$$
V_{F}(y, Y)=\int_{\partial c}\{\exp 2 \pi i y|Y|(\beta(Y), Z)\}(F(Z), n(Z)) d S_{Z} .
$$

Proof of Lemma 2. For each positive integer $q$, let $\Delta_{x}^{q}$ be the $q$ th difference operator in the variable $x$, with difference $z$. I.e., 


$$
\Delta_{x}^{q} g(x, y)=\sum_{j=0}^{q}\left(\begin{array}{l}
q \\
j
\end{array}\right)(-1)^{q-j} g(x+j z, y) .
$$

Then if we set

$$
L_{x}^{q} \hat{J}(x, Y)=\int_{0}^{x} d x_{1} \cdots \int_{0}^{x-1} \hat{J}\left(x_{q}, Y\right) d x_{q} \quad\left(x_{0}=0\right),
$$

we have $I_{x}^{p} \hat{J}(x, Y)=\Delta_{x}^{p} L_{x}^{p} \hat{J}(x, Y)$. So if we can show that $L_{x}^{p} \hat{J}(x, Y)=O\left(F_{2}(x, Y)\right)$, it is clear that the same estimate will hold for $I_{x}^{p} \hat{J}(x, Y)$. We will obtain the desired estimate on $L_{x}^{p} \hat{J}(x, Y)$ as a consequence of the following result:

Suppose $Y \neq 0$ fixed. Then for every integer $q$, satisfying $0 \leqq q \leqq p$, there exists a vector field $G_{q}(Z)=\left(g_{q 1}(Z), \cdots, g_{q n}(Z)\right)$, having the following properties:

A. The functions $g_{q 1}(Z), \cdots, g_{q n}(Z)$ are $C^{w-q-2}$ in $E^{n}$, and on each compact set, their first $w-q-2$ derivatives can be bounded independently of $Y$.

B.

$$
L_{x}^{q} \hat{J}(x, Y)=\frac{w^{q}}{(2 \pi i|Y|)^{q+1}} x^{a(q)} V_{G_{q}}\left(x^{1 / w}, Y\right)
$$

where $a(q)=(n+q w-q-1) w^{-1}$.

Before we pass to the proof of this statement, we note that it immediately gives the desired estimate on $L_{x}^{p} \hat{J}(x, Y)$, if our postulate is applied to $V_{G_{q}}\left(x^{1 / w}, Y\right)$, and $p$ is substituted for $q$.

We proceed now by an induction which terminates at $p$. The assertion is clearly true for $q=0$, since in that case we may take $G_{0}(Z)=\beta(Y)$. Suppose now it is true for some integer $m$, satisfying $0 \leqq m<p$. We will show that this implies its truth for $m+1$.

By hypothesis,

$$
L_{x}^{m+1} \hat{J}(x, Y)=\frac{w^{m}}{(2 \pi i|Y|)^{m+1}} \int_{0}^{x} t^{a(m)} V_{G_{m}}\left(t^{1 / w}, Y\right) d t,
$$

and after a change of variable, this becomes

$$
\frac{w^{m+1}}{(2 \pi i|Y|)^{m+1}} x^{(n+m w+w-m-1) w^{-1}} \int_{0}^{1} t^{n+m w+w-m-2} V_{G_{m}}\left(x^{1 / w} t, Y\right) d t
$$

In order to analyze this integral, we use the following easily verified fact, which is an analogue of the formula for integration in polar coordinates:

Suppose $f(Z)$ is integrable on $C$. Then

$$
\int_{C} f(Z) d V_{Z}=\int_{0}^{1} t^{n-1} d t \int_{\partial C} f(t Z) m(Z) d S_{Z}
$$

where $m(Z)=(Z, n(Z))$. 
(To obtain this formula, it is sufficient to consider only $C^{1}$ homogeneous functions, since their linear combinations are dense in $L^{1}(C)$. For such functions, the result is an immediate consequence of Euler's relation and the divergence theorem.)

Now we can extend $m(Z)$ to be homogeneous of weight 0 in $E^{n}-\{0\}$, by defining $m(Z)=(Z, \operatorname{grad} T(Z))[T(Z)]^{-1 / w}|\operatorname{grad} T(Z)|^{-1}$, and we shall henceforth assume that this extension has been made. Note that with this definition, $m(Z)$ is $C^{\infty}$ in $E^{n}-\{0\}$, and never vanishes there, since $Z$ is never perpendicular to $\operatorname{grad} T(Z)$.

Making use now of (4), we see that (3) can be interpreted as a volume integral

$$
\frac{w^{m+1}}{(2 \pi i|Y|)^{m+1}} x^{(n+m w+w-m-1) w^{-1}} \int_{C} B(Z)\left\{\exp 2 \pi i|Y| x^{1 / w}(\beta(Y), Z)\right\} d V_{Z}
$$

where

$$
B(Z)=[m(Z)]^{-1}[T(Z)]^{(m w+w-m-1) w^{-1}}\left(G_{m}\left([T(Z)]^{-1 / w} Z\right), \frac{\operatorname{grad} T(Z)}{|\operatorname{grad} T(Z)|}\right) .
$$

Now $[T(Z)]^{(m w+w-m-1) w^{-1}}$ is $C^{\infty}$ in $E^{n}-\{0\}$ and homogeneous of weight $m w+w-m-1$, while the product of the remaining factors of $B(Z)$ is homogeneous of weight 0 , and is, by hypothesis, $C^{w-m-2}$ for $Z \neq 0$. We conclude, therefore, that if we define $B(0)=0$, then $B(Z)$ is at least $C^{w-m-2}$ in all of $E^{n}$, since $m w+w-m-2 \geqq w-m-2$.

Thus, by Lemma 3 , there exists a vector field $F(Z)$, such that

1. the components of $F(Z)$ are $C^{w-m-3}$ in $E^{n}$.

2. $\operatorname{div} F(Z)=0$.

3. $(\beta(Y), F(Z))=B(Z)$.

4. on each compact set, the first $w-m-3$ derivatives of the components of $F(Z)$ can be bounded independently of $Y$, since this is true for $B(Z)$.

Now conditions (2) and (3) imply that

$$
\begin{aligned}
\operatorname{div}\left[\left\{\exp 2 \pi i|Y| x^{1 / w}(\beta(Y), Z)\right\} F(Z)\right] \\
\quad=2 \pi i|Y| x^{1 / w} B(Z)\left\{\exp 2 \pi i|Y| x^{1 / w}(\beta(Y), Z)\right\},
\end{aligned}
$$

so by the divergence theorem, (5) is equal to

$$
\frac{w^{m+1}}{(2 \pi i|Y|)^{m+2}} x^{(n+m w+w-m-2) w^{-1}} V_{F}\left(x^{1 / w}, Y\right),
$$

and if we set $G_{m+1}(Z)=F(Z)$, this establishes the desired result for $m+1$. q.e.d.

2. In this section, we will show by an example how in some cases the methods of $\S 1$ can be refined to give considerably sharpened results. We will be working in $E^{2}$, and we will adopt the notation of $\$ 1$, adjusted to the particular case $T(Z)=z_{1}^{2 k}+z_{2}^{2 k}$, where $k$ is a fixed positive integer greater than 1 . Our result 
will be that for this particular choice of $T(Z), R(x)=O\left(x^{(2 k-1) / 4 k^{2}}\right)$, and that this exponent is the best possible. I.e., $R(x)=\Omega\left(x^{(2 k-1) / 4 k^{2}}\right)$.

Definition. For any nonzero point $Y=\left(y_{1}, y_{2}\right)$ in $E^{2}$, set

$$
A(Y)=\min \left(\left|y_{1}\right| /|Y|,\left|y_{2}\right| /|Y|\right) .
$$

LEMMA 4. Let $T(Z)$ be as above. Then for every function $f(Z), C^{1}$ in a neighborhood of $\partial C$, there exists a positive number $M(f)$, depending only on bounds for $f(Z)$ and its first derivatives in a neighborhood of $\partial C$, such that if $Y$ is a point not lying on one of the coordinate axes, then

$$
\left|\int_{\partial C} f(Z) e^{2 \pi i(Y, Z)} d S_{Z}\right| \leqq M(f)|Y|^{-1 / 2}[A(Y)]^{-(k-1) /(2 k-1)} .
$$

Proof. We defer the proof of Lemma 4 to the end of this section.

Now let $\hat{J}(x, Y)$ be defined as before. I.e.,

$$
\hat{J}(x, Y)=\frac{x^{1 / 2 k}}{2 \pi i|Y|} \int_{\partial C} \exp \left\{2 \pi i x^{1 / 2 k}|Y|(\beta(Y), Z)\right\}(\beta(Y), n(Z)) d S_{Z} .
$$

Define $I_{x}^{1} \hat{J}(x, Y)$ as before, except that we now take $z=x^{(3 k-2) / 3 k}$.

Suppose now that $Y$ does not lie on either of the coordinate axes. Then by using Lemma 4, and imitating the proofs of Lemmas 1 and 2, we obtain the following estimates, which are the analogues of the estimates in Lemmas 1 and 2.

1. $I_{x}^{1} \hat{J}(x, Y)=O\left(F_{1}(x, Y)\right)$, where $F_{1}(x, Y)=z x^{1 / 4 k}|Y|^{-3 / 2}[A(Y)]^{-(k-1) /(2 k-1)}$, and the estimate is uniform in $x$ and $Y$.

2. $I_{x}^{1} \hat{J}(x, Y)=O\left(F_{2}(x, Y)\right)$, where $F_{2}(x, Y)=x^{(4 k-1) / 4 k}|Y|^{-5 / 2}[A(Y)]^{-(k-1) /(2 k-1)}$, and the estimate is uniform in $x$ and $Y$.

Suppose now $Y \neq 0$ lies on one of the coordinate axes. Then (6), and a standard application of the method of stationary phase (cf. [1, pp. 51-56]), shows that

(7) $\hat{J}(x, Y)=c_{1} x^{(2 k-1) / 4 k^{2}} \frac{\sin \left(2 \pi|Y| x^{1 / 2 k}-\pi / 4 k\right)}{|Y|^{1+1 / 2 k}}+O\left(x^{(2 k-2) / 4 k^{2}}|Y|^{-(1+1 / k)}\right)$,

for some positive constant $c_{1}$, and the error term is uniform in $x$ and $Y$.

For later use, we define $B(t)$ to be the continuous periodic function

$$
4 c_{1} \sum_{n=1}^{\infty} \frac{\sin (2 \pi n t-\pi / 4 k)}{n^{1+1 / 2 k}} \text {. }
$$

Consider now the family of functions $N_{\varepsilon}(x)(\varepsilon>0)$, defined as in $\S 1$. As before,

$$
\begin{array}{ll}
\lim _{\varepsilon \rightarrow 0} I^{1} N_{\varepsilon}(x) & =I^{1} N(x), \text { and } \\
& I^{1} N_{\varepsilon}(x)=V I^{1} x^{1 / k}+\Sigma^{\prime} \hat{\delta}_{\varepsilon}(N) I_{x}^{1} \hat{\jmath}(x, N) .
\end{array}
$$

We split the last sum into three parts 


$$
\sum_{|N| \leqq x^{1 / 6 k}}^{*} \hat{\delta}_{\varepsilon}(N) I_{x}^{1} \hat{J}(x, N)+\Sigma^{*} \hat{\delta}_{\varepsilon}(N) I_{x}^{1} \hat{J}(x, N)+\underset{|D|>x^{1 / 6 k}}{\sum^{* *}} \hat{\delta}_{\varepsilon}(N) I_{x}^{1} \hat{J}(x, N) .
$$

(Here the single asterisk means that the lattice-points which lie on the coordinate axes are excluded, while the double asterisk means that the sum is taken over precisely those nonzero lattice-points which lie on the coordinate axes.) We estimate the first sum by replacing $I_{x}^{1} \hat{J}(x, N)$ by $F_{1}(x, N)$, the second by replacing $I_{x}^{1} \hat{J}(x, N)$ by $F_{2}(x, N)$, and the third by replacing $\hat{J}(x, N)$ by the estimate in (7). To treat the first and second of the resulting three sums, note that since $\left|\hat{\delta}_{\varepsilon}(N)\right| \leqq 1$ for all $N$ and $\varepsilon>0$, these sums can be dominated by multiples of

and

$$
z x^{1 / 4 k} \int_{0}^{\pi / 4} d \theta \int_{r=1}^{x^{1 / 6 k}} r^{-1 / 2} \theta^{-(k-1) /(2 k-1)} d r
$$

$$
x^{(4 k-1) / 4 k} \int_{0}^{\pi / 4} d \theta \int_{r=x^{1 / 6 k}}^{\infty} r^{-3 / 2} \theta^{-(k-1) /(2 k-1)} d r,
$$

respectively. But $\theta^{-(k-1) /(2 k-1)}$ is integrable over $[0, \pi / 4]$, so both these integrals are finite, and a calculation shows that they are both $O\left(z x^{1 / 3 k}\right)$.

On the other hand, it is clear that as $\varepsilon \rightarrow 0$, the third sum approaches $I^{1} x^{(2 k-1) / 4 k^{2}} B\left(x^{1 / 2 k}\right)+I^{1} O\left(x^{(2 k-2) / 4 k^{2}}\right)$, uniformly on finite $x$-intervals. Thus, letting $\varepsilon \rightarrow 0$, we find that

$$
I^{1} N(x)=V I^{1} x^{1 / k}+O\left(z x^{1 / 3 k}\right)+I^{1} x^{(2 k-1) / 4 k^{2}} B\left(x^{1 / 2 k}\right)+I^{1} O\left(x^{(2 k-2) / 4 k^{2}}\right) .
$$

Now $V I^{1} x^{1 / k}=V z x^{1 / k}+O\left(z x^{1 / 3 k}\right)$, and $N(x)$ is nondecreasing, so

$$
z N(x) \leqq V z x^{1 / k}+O\left(z x^{1 / 3 k}\right)+I^{1} x^{(2 k-1) / 4 k^{2}} B\left(x^{1 / 2 k}\right)+I^{1} O\left(x^{(2 k-2) / 4 k^{2}}\right) .
$$

I.e.,

(8) $N(x)-V x^{1 / k} \leqq \frac{1}{z} I^{1} x^{(2 k-1) / 4 k^{2}} B\left(x^{1 / 2 k}\right)+\frac{1}{z} I^{1} O\left(x^{(2 k-2) / 4 k^{2}}\right)+O\left(x^{1 / 3 k}\right)$,

and this clearly implies that $R(x) \leqq O\left(x^{(2 k-1) / 4 k^{2}}\right)$. Going the other way around,

$$
z N(x+z) \geqq V z x^{1 / k}+I^{1} x^{(2 k-1) / 4 k^{2}} B\left(x^{1 / 2 k}\right)+I^{1} O\left(x^{(2 k-2) / 4 k^{2}}\right)+O\left(z x^{1 / 3 k}\right) .
$$

Now $(x+z)^{1 / k}=x^{1 / k}+O\left(x^{1 / 3 k}\right)$, so

$N(x+z)-V(x+z)^{1 / k} \geqq \frac{1}{z} I^{1} x^{(2 k-1) / 4 k^{2}} B\left(x^{1 / 2 k}\right)+\frac{1}{z} I^{1} O\left(x^{(2 k-2) / 4 k^{2}}\right)+O\left(x^{1 / 3 k}\right)$,

and if we make the substitution $x_{1}=x+z$, we easily obtain that

$$
R\left(x_{1}\right) \geqq O\left(x_{1}^{(2 k-1) / 4 k^{2}}\right) .
$$

I.e., combining results, we find that $R(x)=O\left(x^{(2 k-1) / 4 k^{2}}\right)$, which is a considerable improvement on the estimate $O\left(x^{(2 k-1) / k(4 k-1)}\right)$ which $\$ 1$ gives. We can, moreover, infer from our results that the exponent we have obtained is the best possible. 
To see this, note first of all that $B(t)$ is negative in a neighborhood of $t=0$. If, now, we examine the inequality (8), it is clear that the sum of the second and third terms on the right is $O\left(x^{(2 k-2) / 4 k^{2}}\right)$. Moreover, since the variation of $t^{1 / 2 k}$ over the interval $[x, x+z]$ tends to zero as $x \rightarrow \infty$, it is clear that if $x$ is large, and $x^{1 / 2 k}$ is an integer, then the first term on the right is actually of the order of $x^{(2 k-1) / 4 k^{2}}$, and this suffices to prove the assertion.

Proof of Lemma 4. We shall suppose that $Y$ is in the open first quadrant, and is within $\pi / 4$ radians of the $z_{2}$-axis. This involves no loss of generality, since the techniques required for other cases involve only obvious modifications of the techniques for this case. We shall write $Y$ in polar form as $Y=|Y|\left(y_{1}^{*}, y_{2}^{*}\right)$.

Now there are exactly two points on $\partial C$ at which the normal to $\partial C$ is parallel to $Y$. One of these occurs in the first quadrant. Call it $P=\left(p_{1}, p_{2}\right)$. Then since $\left(p_{1}^{2 k-1}, p_{2}^{2 k-1}\right)$ is the direction of the normal to $\partial C$ at $\left(p_{1}, p_{2}\right)$, we see that $y_{2}^{*} p_{1}^{2 k-1}=y_{1}^{*} p_{2}^{2 k-1}$, or $y_{1}^{*} / y_{2}^{*}=\left(p_{1} / p_{2}\right)^{2 k-1}$. But since $\pi / 4 \leqq \arg Y<\pi / 2$, we have $A(Y)=y_{1}^{*}$, so it is clear that there exists a constant $c_{2}>0$, such that $p_{1} \geqq c_{2}[A(Y)]^{1 /(2 k-1)}$.

Now for purposes of integration, $\partial C$ can be regarded as being composed of two "hemispheres", namely, those points whose $z_{2}$ coordinate is $\geqq 0$, and those points whose $z_{2}$ coordinate is $\leqq 0$. The contribution to the integral in Lemma 4 which comes from the upper hemisphere can be written as

$$
\int_{-1}^{1} m(r) f^{*}(r) e^{2 \pi i|Y| E(r)} d r
$$

where $E(r)=y_{1}^{*} r+y_{2}^{*}\left(1-r^{2 k}\right)^{1 / 2 k}, f^{*}(r)=f\left(r,\left(1-r^{2 k}\right)^{1 / 2 k}\right)$, and $m(r)$ represents the distortion of measure which arises from the projection onto the $z_{1}$-axis.

Suppose now $h(t)$ is a $C^{\infty}$ function, identically 1 in a neighborhood of $t=0$, and having support in an interval $|t| \leqq \varepsilon$, where $\varepsilon$ is a fixed positive number less than $1-(1 / 2)^{1 / 2 k}$. Then the integral in (9) can be written as

$$
\text { (10) } \int_{-1}^{1} h\left(r-p_{1}\right) m(r) f^{*}(r) e^{2 \pi i|Y| E(r)} d r+\int_{-1}^{1}\left(1-h\left(r-p_{1}\right)\right) m(r) f^{*}(r) e^{2 \pi i|Y| E(r)} d r .
$$

To deal with the second integeral, note that if it is combined with the integral which corresponds to it, when the process we have described is duplicated in the lower hemisphere, the result is uniformly $O\left(|Y|^{-1}\right)$ (cf. [6, p. 767]).

Consider now the first integral in (10). (The techniques we will use to estimate this integral apply equally well to the corresponding integral in the lower hemisphere.) The integral in question can be written in the form

$$
\int_{-\varepsilon}^{0} h(r) F(r) e^{2 \pi i|Y| E_{1}(r)} d r+\int_{0}^{\varepsilon} h(r) F(r) e^{2 \pi i|Y| E_{1}(r)} d r,
$$

or 


$$
\int_{0}^{\varepsilon} h(-r) F(-r) e^{2 \pi i|Y| E_{1}(-r)} d r+\int_{0}^{\varepsilon} h(r) F(r) e^{2 \pi i|Y| E_{1}(r)} d r,
$$

where $E_{1}(r)=E\left(r+p_{1}\right)$, and $F(r)=m\left(r+p_{1}\right) f^{*}\left(r+p_{1}\right)$.

We now estimate the second integral in (11). The same technique yields an identical estimate for the first integral. To obtain the desired estimate for the second integral, we need some information about $E_{1}(r)(0 \leqq r \leqq \varepsilon)$. To begin with, we note that it is clear from computation and from the geometrical meaning of $E_{1}(r)$, that for $0 \leqq r \leqq \varepsilon, E_{1}^{\prime}(r), E_{1}^{\prime \prime}(r) \leqq 0$. (Note that $E_{1}(r)$ is simply the component of the vector $\left(r+p_{1},\left(1-\left(r+p_{1}\right)^{2 k}\right)^{1 / 2 k}\right)$ in the direction of the normal to $\partial C$ at $P$.) Again by computation, it is clear that there exists $c_{3}>0$, such that $\left|E_{1}^{\prime \prime}(r)\right| \geqq c_{3}\left(r+p_{1}\right)^{2 k-2}$. On the other hand,

$$
\begin{aligned}
\left|E_{1}^{\prime}(r)\right| & =\int_{0}^{r}\left|E_{1}^{\prime \prime}(t)\right| d t \\
& =\int_{0}^{r / 3}+\int_{r / 3}^{2 r / 3}+\int_{2 r / 3}^{r} .
\end{aligned}
$$

I.e., by the first mean-value theorem for integrals, and the fact that throughout at least one of the three intervals of integration, $\left(r+p_{1}\right)^{2 k-2}$ must be at least of the order of $p_{1}^{2 k-2}$, we obtain the inequality $\left|E_{1}^{\prime}(r)\right| \geqq c_{4} r p_{1}^{2 k-2}$, for some $c_{4}>0$, and hence $\left|E_{1}^{\prime}(r)\right| \geqq c_{5} r[A(Y)]^{(2 k-2) /(2 k-1)}$, for some $c_{5}>0$. (In the case at hand, it is not necessary to split the integral into three parts to obtain the last inequality. A device of this sort is, however, necessary to obtain the corresponding inequality on $\left|E_{1}^{\prime}(-r)\right|$, and this is in turn required to estimate the first integral in (11).)

We now pass to the estimation of the second integral in (11). Set $\delta=|Y|^{-1 / 2}[A(Y)]^{-(k-1) /(2 k-1)}$. Then

$$
\begin{aligned}
\int_{0}^{\varepsilon} h(r) F(r) e^{2 \pi i|Y| E_{1}(r)} d r & =\int_{0}^{\delta}+\int_{\delta}^{\varepsilon} \\
& =O\left(|Y|^{-1 / 2}[A(Y)]^{-(k-1) /(2 k-1)}\right)+\int_{\delta}^{\varepsilon} .
\end{aligned}
$$

(We assume $\delta<\varepsilon$, since otherwise there is nothing to prove.)

Now

$$
\int_{\delta}^{\varepsilon} h(r) F(r) e^{2 \pi i|Y| E_{1}(r)} d r=\frac{1}{2 \pi i|Y|} \int_{\delta}^{\varepsilon} \frac{1}{E_{1}^{\prime}(r)} h(r) F(r) d\left(e^{2 \pi i|Y| E_{1}(r)}\right),
$$

and if we note that $1 / E_{1}^{\prime}(r)$ is increasing,'and apply the second mean-value theorem to the real and imaginary parts of the integral on the right, we immediately obtain that this last expression is $O\left(|Y|^{-1 / 2}[A(Y)]^{-(k-1) /(2 k-1)}\right)$. q.e.d.

\section{REFERENCES}

1. A. Erdélyi, Asymptotic expansions, Dover, New York, 1956. 
2. C. S. Herz, On the number of lattice-points in a convex set, Amer. J. Math. 84 (1962), 126-133.

3. Edmund Hlawka, Über Integrale auf Konvexen Körpern. I, Monatsh. Math. 54 (1950), 1-36.

4. E. Krätzel, Ein Gitterpunktsproblem, Acta Arith. 10 (1964), 215-223.

5. Edmund Landau, Zur analytischen Zahlentheorie der definiten quadratischen Formen, S.-B. Königlich Preuss. Akad. Wiss. 31 (1915), 458-476.

6. Walter Littman, Fourier transforms of surface-carried measures and differentiability of surface averages, Bull. Amer. Math. Soc. 69 (1963), 766-770.

7. J. G. van der Corput, Zahlentheoretische Abschätzungen mit Anwendung auf Gitterpunktprobleme, Math. Z. 17 (1923), 250-259.

YALE UNIVERSITY,

New Haven, Connecticut 